\title{
« The Othello Syndrome » de Uri Caine
}

\section{Jacques Bonnaffé}

Christophe Hausermann (éd.)

\section{OpenEdition}

\section{Journals}

Édition électronique

URL : http://journals.openedition.org/shakespeare/2799

DOI : $10.4000 /$ shakespeare.2799

ISSN : 2271-6424

Éditeur

Société Française Shakespeare

Édition imprimée

Date de publication : 1 mai 2014

Pagination : 19-28

\section{Référence électronique}

Jacques Bonnaffé, « «The Othello Syndrome » de Uri Caine », Actes des congrès de la Société française Shakespeare [En ligne], 31 | 2014, mis en ligne le 01 mai 2014, consulté le 20 avril 2019. URL : http:// journals.openedition.org/shakespeare/2799; DOI : 10.4000/shakespeare.2799 


\title{
«The Othello Syndrome» De Uri Caine
}

\author{
Jacques BONNAFFE
}

$\mathrm{I}$

l n'y a pas de prélude, l'opéra commence immédiatement allegro agitato sur un rapide glissando de cordes menant à un formidable coup de tonnerre qui ébranle la salle. Le rideau s'ouvre à la troisième mesure sur un port de Chypre. Une violente tempête fait rage ! Un chœur attend avec impatience le retour d'Otello, général de la flotte vénitienne et gouverneur de l'île. (Ola ! Bougez pas !)

L'action se passe à Chypre. On est sur le terrain d'emblée! Vous le savez, tout n'est pas simple à Chypre, économie vacillante, rumeur d'un trou sans fond probablement situé sous l'île et qui n'en fait pas un paradis fiscal idéal mais... quand même assez bien niché pour attirer les convoitises ! Et il y a les Turcs sur la vague justement, mais derrière ! Défaits ! Engloutis ! Stermino ! Dispersi ! Distrutti les Turcs ! Les "Ottomans ». Mais ce sont des Turcs, Shakespeare ne se gêne pas pour l'écrire, lui qui serait plutôt porté aux précautions oratoires... dans les Sonnets. Mais je ne vous parle pas des Sonnets! Et nous ne parlons pas de Shakespeare puisque d'emblée nous sommes à Chypre. Autrement nous serions à Venise pour le premier acte. Ah ! Venise c'est autre chose, la Sérénissime, Piazza di San Marco, le Palais des Doges c'était leur Maison Blanche, Washington today. Ne perdons pas de temps, donc laissons tomber Venise, laissons tomber la nuit vénitienne, les rues, Brabantio ! Lamentations d'un père de bonne famille, vénitienne : sa fille Desdemona mariée secrètement à un Arabe, Othello m'a tué ! Même aujourd'hui, ça ne se passerait pas sans histoire. Mais tant mieux, on ne serait pas là non plus, sans histoire. Ce soir nous sommes à Chypre, « le Maure 2 », il a chassé les Turcs, il doit passer la vague. Un Vessilio ! La Déesse demona est avec la foule au port « una Vela una Vela », elle ne crie pas Au voleur Au voleur, non elle attend son heure de liesse dans la liesse générale décrétée pour le retour du vainqueur vénitien. Il frôle le récif, Titanicci, Oricalchi il passe le récif et commande une musique de parade à Verdi pour le retour triomphal au port. 
Fuocco di Gioia ! Colpe di canonne, Una Vela ! Ladro Ladro !

FIRE SONG Bonsoir, Permettez-moi de me présenter Please allow me introduce myself : Iago

The honest Iago, the brave yes the perfect Iago, read Shakespeare, the most honest Iago, that's in it. Yes inhuman dog too, nobody is perfect, slanderer, oui oui Calomniateur...

Iago, sans lui pas d'Othello, j'aurais pu réclamer le titre mais j’ai préféré la discrétion.

Pour la modestie, là personne ne peut me battre.

Enseigne du Général Othello, factotum et videur de pot

Nous ne pouvons être tous maîtres. Et tous les maîtres ne peuvent être loyalement servis

Tu m'avais dit que tu le tenais en haine

J'avais bien dit que je le tenais en haine

Méprisez-moi si je ne le fais pas

Je hais le Maure

mais si j'étais le Maure je ne voudrais pas être Iago

En le servant je ne sers que moi-même

Tu m'avais dit que tu le tenais en haine

J'avais bien dit que je le tenais en haine

Méprisez-moi si je ne le fais pas

Je n'agis ni par amour ni par dévouement mais sous leur apparence dans mon propre intérêt.

Illustration ? Subito : L'heure est aux réjouissances Fuoco di Gioia. Fire Song... Cipriotti et tutti quanti.

FIRE SONG suite Nous laissons de côté le seul personnage triste et grave dans cet ensemble. Roderigo inconsolable petit puceau, viande blanche, amoureux blême de la dame Démona, mais elle n'en sait rien, déclencheur de cette tragédie macabre et sa troisième victime ou quatrième ou cinquième victime selon l'ordre d'importance ou l'heure du décès. Roderigo, qui dans son affliction, trouve pour son malheur un soutien indéfectible en la personne de votre serviteur. 
Tu m'avais dit que tu le tenais en haine

J'avais bien dit que je le tenais en haine Méprisez-moi si je ne le fais pas

Je hais le Maure je te l'ai souvent dit et je te le redis encore et encore. (Attention ce n'est pas parce qu'il est Maure, pas seulement. Je n'ai rien contre les arbres en général. Celui-ci est particulier).

Eviva Othello eviva les revoilà

On pense de par le monde qu'il a rempli ma charge, je ne sais pas si c'est vrai mais moi sur un simple soupçon de ce genre, j'agirais comme sur une certitude

\section{Eviva Othello Othello Othello Othello}

Même si je le hais à l'égal des tourments de l'Enfer je dois hisser le drapeau et l'enseigne de l'amitié

\section{DRINKING SONG}

- Venez Lieutenant Cassio, Beva con me!

- Pas ce soir bon Iago

- J'ai une cruche de vin. Là dehors il y a une bande de vaillants Chypriotes.

- Je voudrais bien que la courtoisie invente un autre rite de célébration

- À la... santé du Noir Othello, inch'Allah.

- J'ai une très pauvre et très malheureuse cervelle pour la boisson

- Oh ce sont des amis, juste une coupe. Je la boirai pour vous ! Beva con me! Prima que svampino!

Ces trois gaillards de Chypre ont l'honneur chatouilleux, maintenant dans ce troupeau d'ivrognes, je dois pousser Cassio à quelque action qui mette l'île en émoi

Beva con me ! Beva con me!

La coupe, la coupe ! del Calice!

Avec une toile d'araignée aussi fine que celle-ci, j’attraperai une aussi grosse mouche que Cassio 
Blanc Cassis ou blanc cassé ? Michael Cassio ! Beva con me ! Beva con me! Non temo il ver...

Beva con me, Non temo il ver de vin de Chiotte ch... Chypriotte si Bevi bevi bevi con me! Bevi bevi con me!

Trinca trinca trincanna...

Avec ce qu'il a bu ce soir il sera plein de querelle et de hargne.

Eviva Eviva Vittoria ! Canto canto et bechier

Et voilà notre capitaine Cassé

Keep up your bright swords, for the dew will rust them

Rengainez-vos épées car la rosée va les rouiller

(sportif)

Othello a dû sanctionner (à regret, la mort dans l'âme) la blessure infligée par le capitaine Cassio au Vénitien Montano qui avait voulu s'interposer dans l'escarmouche avec le jeune Roderigo, entraîné par... par Iago, pour vous servir, l'horlogerie est un de mes passe-temps... Encore que ça... Ça n'était que de l'échauffement.

Men should be what they seem. Or those that be not, would they might seem none.

Voyez son vice.

Les hommes devraient paraître ce qu'ils sont

et ceux qui ne le sont pas, ils devraient ne ressembler à rien

Est-il souvent comme ça?

C'est l'habituel prologue de son sommeil,

Si c'est pas dommage de voir ça mon brave Monsieur Non, c'est une Infirmité, il faut essayer de comprendre

Il peut veiller pendant deux tours d'horloge si la boisson ne balance pas son cerveau, 
Extrait des minutes précieuses dans l'affaire de Monsieur Cassio, Capitaine dégradé, pour avoir provoqué diverses querelles dans l'établissement dit La Taverna con Pergolato, lors de fêtes données pour le retour du général Othello et à l'occasion de ses noces... imprévues... prématurées, précipitées ! Le greffe de ces actes numérotés établi par Monsieur William Shakespeare, Shakespeare, résidant à Stradfordupon-Avon, England

- Qui était-ce que vous poursuiviez de votre épée ? Que vous avait-il fait?

- Le sieur Cassio : Je ne sais pas

- Est-ce possible?

- Je me rappelle une masse de choses mais rien distinctement. Oh Dieu ! Dire que les hommes introduisent un poison dans leur bouche pour qu'il leur dérobe la cervelle. Et dire qu'avec satisfaction nous pouvons nous transformer en bête.

- Allez ! Vous êtes un moralisateur trop sévère. Le bon vin est un bon génie amical quand on en use bien. Puisque la chose est comme elle est, réparez-la à votre avantage. La femme de notre général est maintenant son général (je l'ai entendue cette nuit croyez moi que ça y allait. Elle a mérité son grade, et le Maure a rempli sa charge). Elle est d'un naturel si ouvert, si bon, si obligeant, si heureux. Importunez-la. Confessez-vous ouvertement à elle pour qu'elle vous aide à retrouver votre poste.

- C'est une dame fort exquise

- C'est un morceau de choix. Bien du bonheur pour les draps. Et je vous garantis qu'elle a du tempérament.

- En vérité c'est la plus fraîche et la plus délicate des températures.

- Et quel œil ! Engageant !

— Engageant, mais tout à fait pudique

- Cette querelle entre vous et son mari, suppliez-la de la raccommoder. Cette amitié brisée sera plus forte qu'avant.

- Vous me conseillez bien, honest Iago

- Oui, mais je ne suis pas capitaine, avec mes sabots! 
Je vous assure dans la sincérité de mon amitié et en toute honnête bienveillance, avec mes sabots !

Je serai bien capitaine, avec mes sabots dondaine ho ho ho avec mes sabots !

Ils m'ont appelé vilaine avec mes sabots Je ne suis pas si vilaine avec mes sabots

Puisque le fils du roi m'aime avec mes sabots

S'il le faut je serai reine avec mes sabots

CREDO in un dio crudel che il mia creato

Simile a se // et che nell ire io nomo

Jé crois dans un Dieu crudel qu'il m'a créé, semblable à lui

Dalla vita di un germa o d'un atome (avec un germe et une atome. Crochu !)

Io sono Scelerato... Perché son Uomo

vile son nato - je suis né salaud

comò se dice, une ordure, la fumier,

Io sento il fango originario in me, la fange la merde c'est le mot que je cherchais, the shit,

Si questa, questa e la mia fé

Io con fermo cuore... Qu'on n'est pas prêt de me rattraper, sur ce terrain faut pas me chercher. J'aime le travail bien fait, l'efficacité. Je n'agis ni par amour ni par dévouement mais sous leur apparence, dans mon propre intérêt. Je sais mon prix, je ne méritais pas moins que ce poste, mais mais mais mais, je ne serai pas capitaine et ce n'est pas beau.

Il n'y a pas de remèdes, c'est la plaie du service. L'avancement se fait par recommandation et par faveur et non comme autrefois à l'ancienneté c'est comme ça, c'est comme ça... C'est comme ça Io sono scelerato, c'est comme ça, perche son uomo autrefois le second héritait du premier, 
Io credo que il giusto e un istrion beffardo

le juste est un histrion moqueur

che tutto en lui bugiardo

lagrime bacio squardo sacrificio et honore

Et dopo tanta irisions après tant d'illusions arriva la Morte

et poï et poï... Nulla

La morte e nulla ah ah la mort c'est rien Nulla nulle,

Ecco e Cassio. La saluta e sa vicinna. Madame défendez-moi auprès de votre Seigneur. Petits pas petits. Donnez-moi votre main. Ecco ! Et vos petits doigts souples, comme ils sont durs dites-moi. On les sent prêts pour un toucher vaginal, Aiuuuuuta aiuta Satana, el mio cimento

Othello's Confession

Othello oooo t'es-où Othello Tu fais le Maure ? t'es Hmmm

À quoi tu penses t'es là par terre dans les étoiles

t'es mal tombé ? Tu comptes sur moi pour te relever?

T'es mal tombé, Lion de Venise, je vais pas me salir,

Mon pied sur toi c'est pour t'enfoncer.

Je pourrais t'écraser, si je voulais vraiment t'aider

Io c'est Iago pour vous servir à tout usage et bon à jeter

C'est quoi le Syndrôme

C'est moi le Syndrôme?

Je vais te raconter ce qui va se passer :

$\mathrm{Tu}$ vas pleurer tu vas pleurer,

t'as pas de mouchoir pour t'essuyer, tu l'as donné,

Demande à ta femme de t'éponger, $\mathrm{hmm} \mathrm{hm}$ à qui tu dis?

On agite un mouchoir et déjà t'es fâché,

t'es barré tu vois rouge

Et ça va empirer,

Je te vois névroser grave, graaaaave trop toxique,

Tu vois négro t'es brave, tu t'astiques

Tu peux pas t'arrêter tout le temps d'y penser?

pas pas pas t'arrêter, forcément t'es qu'un mec,

un grand mec : Le Mec! Un pauvre mec

c'est toi le problème, et ça va pas s'améliorer,

(Hmmm hum hum Othello là t'es où ?)

Écoute écoute tu cours à ton propre enfer 
Tu vois dans sa chambre tu vas rentrer, tu vas commander qu'elle se mette à prier.

Comme elle est pas de ta secte des Othellos fêlés, Tu vas lui réciter son Requiem in pace, qu'elle devra répéter :

T'étais où ça hier soir, traîner ? Dans la rue de la gare ?

Et ton mouchoir tu l'as retrouvé ?

Elle va gémir qu'elle l'a paumé Hum hmm hmm ?

Devine qui l'a trouvé ? Tu veux la clé ?

Soit dit en passant comme pour la chambrer :

Cassio tu remets? Qu'est-ce que t'en dis?

T'as qu'à l'amener ! Je l'ai supprimé,

il est trop mort pour la ramener.

Et toi le Maure trop énervé, faut respirer, Pas l'étrangler Othellooo t'es out?

Elle t'a supplié avec des yeux tout amoureux :

un petit mouchoir tu t'es regardé ? C'était déjà fait. Demande d'abord !

Et après seulement tu tues...

Demande autour de toi. Regarde attends juste

toc toc toc c'est ma mégère qui va rentrer,

Tu la connais tu te l'es déjà fait, tu te l'es fait !

Emiliaaa ! Qui va baver qu'elle l'a trouvé,

que c'est à moi qu'elle l'a donné le petit mouchoir,

dans la rue de la gare.

Comment je sais ce qui va se passer?

Parce que je suis là pour raconter, pour y passer

tu as même Cassio qui va rentrer, toujours sur pieds,

innocenté

T'aurais pu l'épargner non ? la sauver,

Non mais quel con, mais quel con ce Lion,

pourquoi pourquoi faut-il toujours d'abord étrangler ?

il te reste plus qu'à te plomber,

arrête, coupe arrête tout, stop, zigouille ça coupe,

oh tu bouges, oh non tu veux pleurer? Tu veux tu peux

Tu peux brailler. T'es retombé bas, si bas

t'es par terre au plus bas

A terra si nel livide Fango 
Même si je le hais à l'égal des tourments de l'Enfer je dois hisser le drapeau et l'enseigne de l'amitié

Titres du concert et du disque de Uri Caine «Syndrome Othello » 1

Othello's Victory

Fire Song

Drinking Son

Love Duet With Othello and Desdemona

Introduction to Act II

Iago's Credo

She's the Only One I Love

Iago's Web

Desdemona's Lament

Am I a Fool?

The Lion of Venice

Othello's Confession

The Willow Song / Ave Maria

Murder

The Death of Othello

Rushdie

Salman Rushdie ("Eleanor's solution", Fury, 2001) : «Othello n'aime pas Desdémone. Ca m'est venu comme ça un jour sans prévenir. Un vrai déclic, une ampoule qui s'allume. Il prétend l'aimer mais ça ne peut pas être vrai. Parce que s'il l'aime alors le meurtre n'a pas de sens. Selon moi, Desdémone est l'épouse trophée d'Othello, son bien le plus précieux et le plus valorisant, la preuve charnelle de sa réputation grandissante dans un monde d'hommes blancs, tu comprends ? C'est la seule chose qu'il aime chez elle. Othello lui-même visiblement n'est pas un Noir mais un Maure : un Arabe, un musulman et son nom sans doute est la latinisation de l'arabe Athalla ou Athullah. Il n'est donc pas une créature du monde chrétien du péché et de la rédemption, mais plutôt de l'univers moral islamique, dont les pôles sont

\footnotetext{
$1_{«}$ Syndrome Othello » se trouve sur ITunes.
} 
l'honneur et la honte. La mort de Desdémone est un cas 'd'honneur vengé par la mort'. Elle n'avait pas besoin d'être coupable. L'accusation suffisait. L'agression subie par sa vertu était incompatible avec l'honneur d'Othello. Voilà pourquoi il ne l'a pas écoutée, pourquoi il ne lui a pas accordé le bénéfice du doute, ni ne lui a pardonné, ni fait ce que tout homme qui aime une femme aurait fait. Othello n'aime que luimême, lui-même en tant qu'amant et chef, ce que Racine écrivain plus ampoulé aurait appelé sa flamme, sa gloire. Pour lui elle n'est même pas une personne. Il l'a réifiée...

En tout cas c'est ce que j'ai essayé de démontrer, et j'ai obtenu mon doctorat peut-être en récompense de mon effronterie, de mon simple culot. » 